\title{
Intersections
}

Canadian Journal of Music

Revue canadienne de musique

\section{Jacotin. 2004. Chansons. Éditées par Frank Dobbins. Centre d'Études Supérieures de la Renaissance, Programme Ricercar. Collection « Épitome musical ». Turnhout: Brepols. xlix, 121 p. ISBN 2-503-51593-2 (couverture souple)}

\section{Patrice Nicolas}

Volume 27, numéro 2, 2007

URI : https://id.erudit.org/iderudit/1013115ar

DOI : https://doi.org/10.7202/1013115ar

Aller au sommaire du numéro

Éditeur(s)

Canadian University Music Society / Société de musique des universités canadiennes

ISSN

1911-0146 (imprimé)

1918-512X (numérique)

Découvrir la revue

Citer ce compte rendu

Nicolas, P. (2007). Compte rendu de [Jacotin. 2004. Chansons. Éditées par Frank Dobbins. Centre d'Études Supérieures de la Renaissance, Programme Ricercar. Collection «Épitome musical ». Turnhout: Brepols. xlix, 121 p. ISBN 2-503-51593-2 (couverture souple)]. Intersections, 27(2), 84-87.

https://doi.org/10.7202/1013115ar

Copyright ( C Canadian University Music Society / Société de musique des universités canadiennes, 2008
Ce document est protégé par la loi sur le droit d'auteur. L'utilisation des services d'Érudit (y compris la reproduction) est assujettie à sa politique d'utilisation que vous pouvez consulter en ligne.

https://apropos.erudit.org/fr/usagers/politique-dutilisation/ 


\section{BooK Reviews / ReCENSIONS}

Jacotin. 2004. Chansons. Éditées par Frank Dobbins. Centre d'Études Supérieures de la Renaissance, Programme Ricercar. Collection "Épitome musical 》. Turnhout : Brepols. xlix, 121 p. ISBN 2-503-51593-2 (couverture souple).

Suffisamment appréciée des éditeurs et des copistes de la Renaissance pour être largement diffusée dans les imprimés et les manuscrits, l’euvre de Jacotin (v.1495-v.1556), digne héritier de Josquin des Prez (v.1450-1521) et Jean Mouton (av.1459-1522), reconnu aujourd'hui comme un maître talentueux et d'une certaine importance, ne bénéficiait jusqualors d'aucune édition critique. Dans la plupart des cas, les quelques éditions modernes existantes de sa musique nëtaient pas conformes aux critères scientifiques que le musicologue de la Renaissance attend aujourd'hui d'une édition savante, notamment la prise en considération de toutes les sources connues pour une même pièce et la présentation, dans un commentaire critique, des variantes musicales et littéraires. Du fait quelles transmettaient les fautes d'impression ou les maladresses du scribe parfois contenues dans la source originale utilisée, ces éditions ne rendaient pas pleinement justice au compositeur. C'est donc à un rigoureux travail dédition musicale critique des quarante-trois chansons de Jacotin auquel sest attaqué Frank Dobbins, de façon à établir un texte musical de qualité dont l'utilité s'impose maintenant à lanalyste comme à l'interprète. Auteur de nombre d'entrées pour le New Grove Dictionary et la Die Musik in Geschichte und Gegenwart, Frank Dobbins cumule plusieurs années dexpérience en enseignement (universités de Londres, Tours et Montréal notamment) et collabore régulièrement avec certains ensembles musicaux dont les King's Singers. Spécialiste des rapports entre musique, thêâtre et poésie à la Renaissance, il est lauteur de plusieurs monographies et éditions musicales, notamment Music in Renaissance Lyons, The Oxford book of French chansons et Charles Tessier : Airs, Villanelles and Lute Songs.

En guise d'introduction, léditeur s'emploie à identifier ce compositeur mystérieux mieux connu sous le diminutif amical du prénom Jacques (Jacotin), situation courante chez les musiciens français et flamands de la Renaissance. Lutilisation exclusive de ce diminutif dans les sources manuscrites et imprimées a amené plus d'un musicologue à confondre le véritable Jacotin avec les compositeurs Jacob Godebrye alias Jacotyn ( $\dagger 1529)$, Jacquet de Berchem (v.1505-1567), Jacques Colebault alias Jacquet de Mantoue (1483-1559) et Jacques Arcadelt (?1507-1568). Une étude approfondie des documents darchives permet cependant à Dobbins de pousser plus avant les hypothèses de ses prédécesseurs, affirmant que le Jacotin des sources musicales doit selon toute vraisemblance être identifié avec le compositeur Jacques Frontin alias Jacotin Lebel originaire de Normandie. Ce dernier fut chantre de la chapelle particulière du pape Léon X (1475-1521, sed. 1513-1521) à Rome, puis membre de 
la chapelle royale de France sous François $I^{\text {er }}$ (1494-1547, reg. 1515-1547) et Henri II (1519-1559, reg. 1547-1559).

Comme toute édition critique qui se veut rigoureuse, cette étude des chansons de Jacotin ne se contente pas de donner que la seule édition du conducteur de l’œuvre musicale réalisée d'après les parties séparées de la source principale utilisée. Léditeur a pris soin de faire accompagner la partition de divers éléments, tels la liste des attributions (parfois contradictoires) au(x) compositeur(s) et un relevé minutieux des variantes musicales et littéraires, qui permettent de retracer la genèse de l'œuvre grâce à létude des sources et leur collationnement. Toutes ces informations sont rassemblées dans un commentaire critique historicisant l'œuvre, dans lequel nous trouvons une présentation des détails et des sources annexes. Une à une, les quarante-trois chansons de Jacotin nous sont présentées. Suivant la présentation du texte littéraire en moyen français tel qu'il se retrouve dans la source originale ainsi que sa traduction anglaise littérale mais consonante avec l'articulation du discours musical, se trouve la liste complète des sources musicales ayant assuré la transmission de cette œuvre particulière. Par l'étude de ces sources et en s'appuyant sur nombres d'ouvrages musicologiques, Dobbins résout les quelques problèmes d'attributions contradictoires ou conflictuelles, comme par exemple dans Aupres de vous attribuée à Claudin de Sermisy par Daniel Heartz sur la base de sa perfection et de quelques imprimés tardifs. Toutefois, Dobbins démontre brillamment, d'une part, que Pierre Attaingnant, imprimeur du roi qui connaissait les deux compositeurs, associa ce motet à Jacotin dès c.1528 (Trente et quatre chansons musicales a quatre parties, RISM [c.1528]), d'autre part que les gestes cadentiels précédés d'une harmonie de sous-dominante et enrichis de syncopes et retards dissonants présents dans cette œuvre caractérisent le style jacotinien mais ne se retrouvent pas chez Sermisy.

Les variantes musicales et littéraires présentes parmi les autres sources, ainsi que les amendements apportés par l'éditeur sont pour leur part rigoureusement notés, et la forme musicale de même que celle du texte littéraire soigneusement analysées, ce qui laisse entrevoir les vastes connaissances de l'éditeur sur la poétique médiévale et renaissante des grands rhétoriqueurs. De plus, lorsqu'il a été en mesure de retracer une version du texte littéraire plus élaborée que celle utilisée par Jacotin (comme dans Vostre beaulté et Hellas pourquoy notamment), le musicologue a fourni les strophes additionnelles de manière à permettre une interprétation strophique plus longue bien qu'il n'y ait " aucune preuve que Jacotin adopta la forme strophique du voix de ville même tard dans sa carrière, pas plus qu'il ne le fit pour l'ode et le sonnet " (p. xiv). Bien que Dobbins ne soit pas le premier à procéder de la sorte, aucune autre édition moderne de chansons ne propose des strophes additionnelles de façon aussi systématique. Malheureusement, la paternité de ces textes littéraires le plus souvent de nature courtoise mais aussi grivoise et rustique demeure dans la plupart des cas incertaine. Seuls quelques-uns peuvent être attribués avec un certain degré de certitude aux poètes Mellin de Saint-Gelais (v.1487-1558, Voyant souffrir et $\mathrm{Si}$ pour aymer) et Jean Marot (?1450-1526, A tout jamais). 
Une analyse très fouillée des mélodies de Jacotin a également permis à Dobbins de démontrer l'utilisation de cantus prius facti. Par exemple, comme le note l'éditeur, le Tenor de Hellas pourquoy reproduit presqu'intégralement la mélodie qui accompagne le même texte dans le chansonnier monophonique dit "de Bayeux " (Paris, BnF, Ms. Fr. 9346), alors que dans la chanson à 3 voix Je suis desheritée, Jacotin composa deux nouvelles voix pour accompagner le Superius emprunté à un modèle à quatre voix attribué tantôt à Lupus (fl. 1518-30), tantôt à Pierre Cadéac (fl. 1538-56). Enfin, en ce qui a trait à loordre de succession des chansons, celles-ci sont présentées selon l'effectif vocal qu'elles utilisent (i.e. quatre, trois puis deux voix) et un classement chronologique de deuxième niveau, selon la première source publiée, s'opère à l'intérieur de chacun de ces trois regroupements. Cela offre au lecteur une présentation homogène des chansons qu'un ordonnancement par ordre alphabétique ou uniquement chronologique - faisant se succéder des œuvres au nombre de voix différent - n'aurait permis.

Somme toute, et à l'image de la New Josquin Edition, l'édition des chansons de Jacotin est impeccable et le musicologue autant que l'interprète y trouveront leur compte. Elle nous présente l'œuvre d'un compositeur talentueux et dont la technique contrapuntique stylisée et élégante (Contentement, $A$ tout jamais, Vostre beaulté, D'ung desplaisir, entre autres) n'est pas sans rappeler Josquin et Mouton. Le seul reproche que l'on peut faire à son éditeur est d'avoir opéré une diminution de moitié des valeurs de notes et de silences, transcrivant une brève en ronde, une semi-brève en blanche, et ainsi de suite. Cette façon de faire, préconisée notamment par Willi Apel dans les années 1940, s'appuyait sur le fait que l'unité de pulsation fut représentée au fil des époques (successivement) par la longue (1200-1250), la brève (1250-1300), la semi-brève (1300-1450), la minime (1450-1600) et enfin la semi-minime, notre noire moderne (1600 à aujourd'hui). Or, le caractère essentiel de la notation originale écope beaucoup de cette façon de faire et de nombreux musicologues préfèrent aujourd'hui conserver les valeurs originelles.

Cette dernière critique naltère en rien la qualité de cette édition brillamment réalisée et qui répond à l'un des besoins les plus urgents de la musicologie et de l'étude de la musique ancienne, à laquelle il importe de connaître sans retard et dans son intégralité l'œuvre encore partiellement inédite de ce maitre important de la Renaissance que fut Jacotin. Et si l'un des projets non réalisés d'Henry Expert, dont le nom est toujours "synonyme de foi et de conscience artistique ", était d'inclure dans sa série Les Maîtres Musiciens de la Renaissance Française une édition moderne des chansons de Jacotin (p. v) , il est fort à parier qu'il aurait approuvé celle que nous propose aujourd'hui Frank Dobbins.

\section{RÉFÉRENCES}

Apel, Willi. 1942. The notation of polyphonic music, 900-1600. Cambridge : Mediaeval Academy of America. 
Bernstein, Lawrence F., dir. 1984. La couronne et fleur des chansons a troys. Masters and Monuments of the Renaissance, vol. 3. New York: The Broude Trust.

Brobeck, John Thomas. 1991. "The motet at the court of Francis I ". Thèse de doctorat, University of Pennsylvania.

Cazaux, Christelle. 2002. La Musique à la Cour de François $I^{e r}$. Mémoires et documents de l'école des Chartes, $\mathrm{n}^{\circ} 65$. Centre d'Études Supérieures de la Renaissance, collection "Épitome musical ». Paris : École Nationale des Chartes - Programme " Ricercar ".

Dobbins, Frank, dir. 1987. The Oxford book of French chansons. Oxford et Toronto : Oxford University Press.

—. 1992. Music in Renaissance Lyons. Collection "Oxford monographs on music ». Oxford : Clarendon Press; New York: Oxford University Press.

$\longrightarrow$ dir. 2006. Charles Tessier : Airs, Villanelles and Lute Songs. Collection "Épitome musical ». Turnhout : Brepols Publishers.

Elders, Willem et al., dir. 1994-. New Josquin Edition : The collected works of Josquin Des Prez. Utrecht : Koninklijke Vereniging voor Nederlandse Muziekgeschiedenis.

Expert, Henry, dir. [s.d.]. Les Maîtres musiciens de la Renaissance française. Réimpression de l'édition originale (Paris, 1894-1908). New York : Broude Brothers.

F. R. 1952. « Henry Expert (1863-1952) ». Revue de musicologie 34, n 103/104 : 169.

Gehrenbeck, David Maulsby. 1970. « Motetti de la corona : A study of Ottaviano Petrucci's four last-known motet prints (Fossombrone, 1514, 1519), with 44 transcriptions ». Thèse de doctorat, Union Theological Seminary in the city of New York.

Giraud, Yves, dir. 1965. La Fleur des chansons rustiques de la Renaissance française. Collection "A Coeur Joie ». Paris : Les presses d'Ile-de-France.

Hewitt, Helen, dir. [1967]. Canti B, numero cinquanta, Venice, 1502. Monuments of Renaissance music, vol. 2. Chicago : University of Chicago Press.

-1978. Harmonice musices odhecaton A / Petrucci. Studies and documents (Mediaeval Academy of America), $\mathrm{n}^{0}$ 5. New York : Da Capo Press.

Lesure, François, dir. [1953]. Anthologie de la Chanson Parisienne. Avec la collaboration de N. Bridgman, I. Cazeaux, M. Levin, K. J. Levy et D. P. Walker. Monaco : Éditions de l'Oiseau-Lyre.

Picker, Martin, dir. 1987. The Motet Books of Andrea Antico. Monuments of Renaissance Music, vol. 8. Chicago et Londres : The University of Chicago Press.

Sherr, Richard, dir. 1992. Selections from Motetti de la corona [libro secundo] (Fossombrone, 1519), Motetti de la corona [libro tertio] (Fossombrone, 1519), Motetti de la corona [libro quarto] (Fossombrone, 1519). Sixteenth-Century Motet, vol. 5. New York: Garland Publishers. 